\title{
Ergodicity of the 2-D Navier-Stokes Equation Under Random Perturbations
}

\author{
Franco Flandoli ${ }^{1}$, Bohdan Maslowski ${ }^{2}$ \\ ${ }^{1}$ Scuola Normale Superiore, Piazza dei Cavalieri 7, I-56100 Pisa, Italy \\ 2 Mathematical Institute, Czech Academy of Sciences, Žitná 25, 11567 Prague 1, Czech Republic
}

Received: 30 April 1994

\begin{abstract}
A 2-dimensional Navier-Stokes equation perturbed by a sufficiently distributed white noise is considered. Existence of invariant measures is known from previous works. The aim is to prove uniqueness of the invariant measures, strong law of large numbers, and convergence to equilibrium.
\end{abstract}

\section{Introduction}

Consider a viscous incompressible fluid in a bounded domain. The following ergodic principle lies at the foundation of the statistical approach to the fluid dynamic (see $[17,23]$ ): there exists an equilibrium measure $\mu$ over the phase space (a space of velocity fields) such that, for every regular observable defined over the phase space, and for every initial velocity field (except for a set of initial fields that is negligible in some sense), the time average of the observable tends, as time goes to infinity, to the mean value of the observable with respect to $\mu$. A rigorous justification of this principle is not known. The aim of this paper is to prove this result in the case of the 2-dimensional Navier-Stokes equation perturbed by a sufficiently distributed white noise. The existence of invariant measure for such equation has been already known (cf. $[23,4,11,12]$, under different conditions). Here it is proved, under proper assumptions on the noise, that the invariant measure is unique, it satisfies a strong law of large numbers, and the convergence to equilibrium takes place.

In the case of finite dimensional differential equations, it is well known that a non-degenerate white noise perturbation yields the previous ergodic results. However, the analysis of this problem in the infinite dimensional case is considerably more difficult, and only the recent development of suitable techniques (cf. for instance $[7,15,16,19,20,21,3,6,8])$ gave the possibility to prove the result for Navier-Stokes equation. Some of the restrictions that we have to impose on the noise are quite standard compared with the current literature on ergodicity of infinite dimensional equations, but it is reasonable to expect that they could be removed by future improvements of the methods. 


\section{Preliminaries on the Navier-Stokes Equation}

Let $D$ be a bounded open domain of $\mathbf{R}^{2}$ with regular boundary $\partial D$. We consider the 2-dimensional stochastic Navier-Stokes equation in $D$ for an incompressible fluid flow:

$$
\frac{\partial u(t, \xi)}{\partial t}+(u(t, \xi) \cdot \nabla) u(t, \xi)-\Delta u(t, \xi)+\nabla p(t, \xi)=f(\xi)+n(t, \xi),
$$

$t \in[0, T], \xi \in D$, subject to the incompressibility condition

$$
\operatorname{div} u(t, \xi)=0, \quad t \in[0, T], \xi \in D,
$$

the boundary condition

$$
u(t, \xi)=0, \quad t \in[0, T], \xi \in \partial D,
$$

and the initial condition

$$
u(0, \xi)=u_{0}(\xi), \quad \xi \in D
$$

For simplicity, we have written the equation in dimensionless form, and with the physical constants (density and viscosity) equal to 1 . Here $n(t, \xi)$ is a Gaussian random field, white noise in time, satisfying the conditions imposed below. Definitions and assumptions concerning this equation will be given at the level of the classical abstract formulation that we are going to introduce.

Note that we use the symbol $\xi$ for the spatial variable in the formal equation (1) while symbols like $x, y, z$, etc. are reserved for elements of the Hilbert spaces, in the framework of which the abstract counterpart of (1) is introduced.

Let $\mathscr{V}$ be the space of infinitely differentiable 2-dimensional vector fields $u(\xi)$ on $D$ with compact support strictly contained in $D$, satisfying $\operatorname{div} u(\xi)=0$. We denote by $V_{\alpha}$ the closure of $\mathscr{V}$ in $\left[H^{\alpha}(D)\right]^{2}$, for $\alpha \geqq 0$, and we set in particular

$$
H=V_{0}, \quad V=V_{1} .
$$

We denote by $|$.$| and \langle.,$.$\rangle the norm and inner product in H$. Identifying $H$ with its dual space $H^{\prime}$, and identifying $H^{\prime}$ with a subspace of $V^{\prime}$ (the dual space of $V$ ) we have $V \subset H \subset V^{\prime}$, and we can denote the dual pairing between $V$ and $V^{\prime}$ by $\langle.,$.$\rangle when no confusion may arise.$

Moreover, we set $D(A)=\left[H^{2}(D)\right]^{2} \cap V$, and define the linear operator $A$ : $D(A) \subset H \rightarrow H$ as $A u=-P \Delta u$, where $P$ is the projection from $\left[L^{2}(D)\right]^{2}$ to $H$. Since $V$ coincides with $D\left(A^{1 / 2}\right)$, we can endow $V$ with the norm $\|u\|=\left|A^{1 / 2} u\right|$. The operator $A$ is positive selfadjoint with compact resolvent; we denote by $0<\lambda_{1} \leqq \lambda_{2} \leqq \cdots$ the eigenvalues of $A$, and by $e_{1}, e_{2}, \ldots$ a corresponding complete orthonormal system of eigenvectors. We remark that $\|u\|^{2} \geqq \lambda_{1}|u|^{2}$.

We define the bilinear operator $B(u, v): V \times V \rightarrow V^{\prime}$ as

$$
\langle B(u, v), z\rangle=\int_{D} z(\xi) \cdot(u(\xi) \cdot \nabla) v(\xi) d \xi
$$

for all $z \in V$. By the incompressibility condition we have

$$
\langle B(u, v), v\rangle=0, \quad\langle B(u, v), z\rangle=-\langle B(u, z), v\rangle .
$$



tion

In place of Eqs. (1)-(4) we shall consider the abstract stochastic evolution equa-

$$
\left\{\begin{array}{l}
d u(t)+A u(t) d t+B(u(t), u(t)) d t=f d t+G d w(t), \quad t \in[0, T] \\
u(0)=u_{0}
\end{array}\right.
$$

Here we assume that $f \in H$ (this can be relaxed in some of the next statements, but we omit this level of generality), $w(t)$ is a cylindrical Wiener process in $H$ (cf. [9]) defined on a complete probability space $(\Omega, \mathscr{F}, P)$, with expectation denoted by $E$, and

(A1) $G: H \rightarrow H$ is a bounded linear operator, injective, with range $\mathscr{R}(G)$ dense in $H$ and satisfying

$$
D\left(A^{\frac{1}{2}}\right) \subset \mathscr{R}(G) \subset D\left(A^{\frac{3}{8}+\varepsilon}\right)
$$

for some $\varepsilon>0$.

Assumption (A1) is the basic condition on the noise that we have to impose in order to obtain the main ergodic result of the paper. Some preliminary results will hold true under more general conditions, indicated later by (A2), etc., that will be stated when it is necessary.

We shall denote by $\mathscr{F}_{S}$ the $\sigma$-algebra generated by $w(\tau)$ for $\tau \leqq s$.

Remark 2.1. An example of the noise satisfying condition (A1) is

$$
n(t, .)=G \frac{d w(t)}{d t}=\sum_{n=1}^{\infty} \sigma_{n} \frac{d \beta_{n}(t)}{d t} e_{n}
$$

where $\left\{\beta_{n}\right\}$ is a sequence of independent 1-dimensional Wiener processes, and $\left\{\sigma_{n}\right\}$ satisfies

$$
c n^{-\frac{1}{2}} \leqq \sigma_{n} \leqq C n^{-\frac{3}{8}-\varepsilon} \quad \forall n \in \mathbf{N}
$$

for some constants $c, C>0$. It is so because the eigenvalues $\lambda_{n}$ of the Stokes operator $A$, in 2-space dimension, behave like $n$ (cf. [22]).

Remark 2.2. Another example of operator $G$ satisfying (A1) is

$$
G=A^{-\gamma} L
$$

with $L$ an isomorphism in $H$ and

$$
\frac{3}{8}<\gamma \leqq \frac{1}{2}
$$

Let us summarize some definitions and results concerning Eq. (5). The main results of the paper will be given in Sect. 3 .

2.1. Existence, Uniqueness and Regularity. A classical definition of solution to Eq. (5) would require, among other properties, that the trajectories of $u$ are square integrable in time with values in $V$. This condition is too strong in the present context, due to the low regularity of the noise. We shall adopt the following natural (although not classical) definition of solution. This definition and the subsequent results are discussed in more details in [11]. 
Definition 2.1. We say that a stochastic process $u(t, \omega)$ is a generalized solution of Eq. (5) if

$$
u(., \omega) \in C([0, T] ; H) \cap L^{2}\left(0, T ; D\left(A^{\frac{1}{4}}\right)\right)
$$

for $P$-a.e. $\omega \in \Omega, u$ is progressively measurable in these topologies, and P-a.s. Eq. (5) is satisfied in the integral sense

$$
\begin{gathered}
\langle u(t), \phi\rangle+\int_{0}^{t}\langle u(s), A \phi\rangle d s-\int_{0}^{t}\langle B(u(s), \phi), u(s)\rangle d s \\
=\left\langle u_{0}, \phi\right\rangle+\int_{0}^{t}\langle f, \phi\rangle d s+\left\langle w(t), G^{*} \phi\right\rangle
\end{gathered}
$$

for all $t \in[0, T]$ and all $\phi \in D(A)$.

Remark 2.3. Since

$$
|\langle B(u(s), \phi), u(s)\rangle| \leqq C\|\phi\||u(s)|_{L^{4}}^{2} \leqq C\|\phi\||u(s)|_{D\left(A^{\frac{1}{4}}\right)}^{2}
$$

the integral term with $B$ is well defined. Moreover, since formally

$$
\langle B(u(s), \phi), u(s)\rangle=-\langle B(u(s), u(s)), \phi\rangle
$$

we see that the integral equation corresponds to Eq. (5).

Consider the auxiliary Ornstein-Uhlenbeck equation

$$
\left\{\begin{array}{l}
d z(t)+A z(t) d t=G d w(t) \quad t \geqq 0 \\
z(0)=0
\end{array}\right.
$$

This equation has a unique progressively measurable solution with $P$-a.e. trajectory $z(., \omega) \in D\left(A^{\frac{1}{4}}\right)$ under assumption (A2) below, and $z(., \omega) \in D\left(A^{\frac{3}{8}}\right)$ under (A3) (see [9], Theorem 5.16).

Equation (5) is studied by the change of variable $v(t)=u(t)-z(t)$. This is the reason for the uniqueness statement of the following theorem; uniqueness in the case (i) below is an open problem. On $G$ we need to impose weaker conditions than (A1). We shall need one of the following conditions:

(A2) $G: H \rightarrow H$ is a bounded linear operator, with range $\mathscr{R}(G)$ satisfying

$$
\mathscr{R}(G) \subset D\left(A^{\frac{1}{4}+\varepsilon}\right)
$$

for some $\varepsilon>0$;

(A3) $G: H \rightarrow H$ is a bounded linear operator, with range $\mathscr{R}(G)$ satisfying

$$
\mathscr{R}(G) \subset D\left(A^{\frac{3}{8}+\varepsilon}\right)
$$

for some $\varepsilon>0$.

Clearly $(\mathrm{A} 1) \Rightarrow(\mathrm{A} 3) \Rightarrow(\mathrm{A} 2)$. 
Theorem 2.1. Assume that condition (A2) is satisfied. Then

i) For each initial condition $u_{0} \in H$, there exists a generalized solution $u$ of Eq. (5) satisfying

$$
u(., \omega) \in C([0, T] ; H) \cap L^{2}\left(0, T ; D\left(A^{\frac{1}{4}}\right)\right)
$$

for $P$ a.e. $\omega \in \Omega$.

ii) One and only one of such solutions satisfies the additional property:

$$
u(., \omega)-z(., \omega) \in L^{2}(0, T ; V)
$$

for $P$ a.e. $\omega \in \Omega$; in the sequel, all the statements will be referred to this canonical solution.

iii) The process $u$ defined in part ii) is a Markov process in $H$, and it satisfies the Feller property.

iv) Assume now that condition (A3) is satisfied. If in addition $u_{0} \in D\left(A^{\frac{1}{4}}\right)$, then

$$
u(., \omega) \in C\left([0, T] ; D\left(A^{\frac{1}{4}}\right)\right) \cap L^{2}\left(0, T ; D\left(A^{\frac{3}{8}}\right)\right)
$$

for $P$ a.e. $\omega \in \Omega$; moreover, for every $t_{0} \in(0, T)$, and every $u_{0} \in H$ the corresponding solution satisfies

$$
u(., \omega) \in C\left(\left[t_{0}, T\right] ; D\left(A^{\frac{1}{4}}\right)\right)
$$

for $P$ a.e. $\omega \in \Omega$.

This theorem is a particular case of a result proved in [11] (except that the operator $G$ was diagonal with $A$ in that paper, but the proof in the present case is the same). Anyway, the main ingredients for the proof will be derived in the Appendix for independent purposes, so that the reader may easily complete the proof, along classical lines (cf. [2, 22, 23]).

2.2. Invariant Measures. Let $P_{t}$ be the Markov semigroup in the space $C_{b}(H)$ associated to the stochastic Navier-Stokes equation (5), defined as

$$
\left(P_{t} \phi\right)(x)=E \phi(u(t, x)), \quad \phi \in C_{b}(H),
$$

where $u(t ; x)$ is the solution of Eq. (5) (given by Theorem 2.1 (ii)) with initial condition $x \in H$. This defines a dual semigroup $P_{t}^{*}$ in the space $\operatorname{Pr}(H)$ of probability measures on $H$ :

$$
\int_{H} \phi d\left(P_{t}^{*} v\right)=\int_{H} P_{t} \phi d v
$$

for all $\phi \in C_{b}(H)$ and $v \in \operatorname{Pr}(H)$. A measure $\mu \in \operatorname{Pr}(H)$ is called invariant if $P_{t}^{*} \mu=\mu$, or equivalently if

$$
\int_{H} \phi d \mu=\int_{H} P_{t} \phi d \mu
$$

for all $t \geqq 0, \phi \in C_{b}(H)$. The invariant measures are, in other words, the equilibrium measures of the Markov process. 
In [11] it has been proved (see [12] for a more general result):

Theorem 2.2. Under the assumption (A2), the stochastic Navier-Stokes equation (5) has an invariant measure.

\section{Main Result}

Recall that $u\left(t ; u_{0}\right)$ stands for the solution (given by Theorem 2.1, (ii)) of Eq. (5) with initial condition $u(0)=u_{0}$. The aim of this paper is to prove:

Theorem 3.1. Assume that condition (A1) is fulfilled. Then:

i) the stochastic Navier-Stokes equation (5) has a unique invariant measure $\mu$;

ii) for all $u_{0} \in H$ and all Borel measurable functions $\phi: H \rightarrow \mathbf{R}$, such that $\int_{H}|\phi| d \mu<\infty$,

$$
\lim _{T \rightarrow \infty} \frac{1}{T} \int_{0}^{T} \phi\left(u\left(t ; u_{0}\right)\right) d t=\int_{H} \phi d \mu
$$

P-a.s.;

iii) for every Borel measure $v$ on $H$ we have that

$$
\left\|P_{t}^{*} v-\mu\right\|_{v} \rightarrow 0 \text { as } t \rightarrow \infty
$$

where $\|.\|_{v}$ stands for the total variation of a measure. In particular, it follows that

$$
P_{t}^{*} v(B) \rightarrow \mu(B) \text { as } t \rightarrow \infty
$$

for every Borel set $B \subset H$.

To prove this theorem we use the following general results. Denote by $P(t, x,$. the transition probability measure defined as

$$
P(t, x, B)=P_{t}^{*} \delta_{x}(B)=P(u(t ; x) \in B)
$$

for $t \geqq 0, x \in H$ and $B \in \mathscr{B}(H)$.

Theorem 3.2. Assume that the probability measures $P(t, x,),. t>0, x \in H$, are all equivalent, in the sense that they are mutually absolutely continuous. Then properties i), ii) and iii) of Theorem 3.1 hold true.

Parts (i), (ii), and the weaker version (7) of (iii), have been proved essentially by Doob [10]; see also Khas'minskii [14]. The part (iii) is due to Seidler [20], Proposition 2.5, and Stettner [21], Theorem 1.

It is known that irreducibility and strong Feller property imply the equivalence of the measures $P(t, x$, ) (cf. [14]). By irreducibility we mean that for all $t>0$, all $x, y \in H$, and all $\rho>0$, denoting by $B(y, \rho)$ the ball in $H$ of center $y$ and radius $\rho$, we have $P\{u(t ; x) \in B(y, \rho)\}>0$. By strong Feller property we mean that $P_{t}$ can be extended to a continuous operator from $B(H)$ to $C_{b}(H)$, where $B(H)$ is the space of bounded measureable functions on $H$. In the case of the stochastic Navier-Stokes equation we can prove the irreducibility in $H$, even in a stronger sense, but we cannot prove the strong Feller property in $H$. On the other side, we can prove the strong Feller property in $D\left(A^{\frac{1}{4}}\right)$, even in a stronger sense, but not 
the irreducibility in $D\left(A^{\frac{1}{4}}\right)$. Then we have to merge carefully the special kind of irreducibility and strong Feller property that we can prove, to obtain the equivalence of the measures $P(t, x,$.$) . This requires a new result on the equivalence problem,$ that is proved in the next section. The following Sects. 5 and 6 will be devoted to the proof of the particular irreducibility and strong Feller properties available for the Navier-Stokes equation.

\section{A Sufficient Condition for Equivalence of Transition Probabilities}

In this section we work under the assumption (A3), but notice that Theorem 4.1 below is a general statement, independent of the particular framework of the NavierStokes equation.

For $y \in D\left(A^{\frac{1}{4}}\right), \varepsilon, M>0$, let

$$
U(y, \varepsilon, M)=\left\{z \in D\left(A^{\frac{1}{4}}\right) ;|z-y|<\varepsilon,\left|A^{\frac{1}{4}} z\right| \leqq M\right\} .
$$

We introduce the following version of the irreducibility property:

(I) For all $x, y \in D\left(A^{\frac{1}{4}}\right)$ there exists $M>0$ such that for all $\varepsilon>0$ :

$$
P(t, x, U(y, \varepsilon, M))>0
$$

for every $t>0$.

Moreover, we introduce the following version of the strong Feller property:

(SF) For all $\Gamma \in \mathscr{B}\left(D\left(A^{\frac{1}{4}}\right)\right.$ ) (the Borel $\sigma$-algebra of $D\left(A^{\frac{1}{4}}\right)$ ), all $t>0$, and all $x_{n}, x \in D\left(A^{\frac{1}{4}}\right)$ such that $x_{n} \rightarrow x$ in $H$ and $\left|A^{\frac{1}{4}} x_{n}\right| \leqq C$ for some constant $C>0$, it holds

$$
P\left(t, x_{n}, \Gamma\right) \rightarrow P(t, x, \Gamma)
$$

Condition (I) implies the irreducibility in $H$ but not in $D\left(A^{\frac{1}{4}}\right)$. On the other hand, condition (SF) implies the strong Feller property in $D\left(A^{\frac{1}{4}}\right)$ but not in $H$.

For the sequel, note that the elements of the set $\mathscr{B}\left(D\left(A^{\frac{1}{4}}\right)\right)$ are the intersections of Borel sets of $H$ with $D\left(A^{\frac{1}{4}}\right)$.

The following result is general, and does not depend on the fact that $P(t, x,$. arises from the Navier-Stokes equation.

Theorem 4.1. Let $P(t, x,),. t>0$ and $x \in D\left(A^{\frac{1}{4}}\right)$, be a family of Markov transition probabilities on $D\left(A^{\frac{1}{4}}\right)$, satisfying conditions $(\mathrm{I})$ and $(\mathrm{SF})$. Then the distributions $P(t, x,$.$) are equivalent, for all t>0$ and $x \in D\left(A^{\frac{1}{4}}\right)$.

Proof. Assume $P\left(t, x_{0}, \Gamma\right)>0$ for some $t>0, x_{0} \in D\left(A^{\frac{1}{4}}\right), \Gamma \in \mathscr{B}\left(D\left(A^{\frac{1}{4}}\right)\right)$. Let $s>t, x \in D\left(A^{\frac{1}{4}}\right)$ be given. Let us prove that $P(s, x, \Gamma)>0$. By (SF), for each $M>0$ there exists $\varepsilon>0$ such that $P(t, y, \Gamma)>0$ for all $y \in U\left(x_{0}, \varepsilon, M\right)$. Take for this $M$ the one from (I) (corresponding to $x=x, y=x_{0}, t=s-t$ ). So we have that for some $M>0$ and $\varepsilon>0$,

$$
P\left(s-t, x, U\left(x_{0}, \varepsilon, M\right)\right)>0
$$


and

$$
P(t, y, \Gamma)>0
$$

for $y \in U\left(x_{0}, \varepsilon, M\right)$. Now, by the Chapman-Kolmogorov equation, we obtain

$$
P(s, x, \Gamma) \geqq \int_{U\left(x_{0}, \varepsilon, M\right)} P(t, y, \Gamma) P(s-t, x, d y)>0,
$$

so we have proved that $P(s, x, \Gamma)>0$ holds for any $x \in D\left(A^{\frac{1}{4}}\right)$ and $s>t$.

If $s<t$, take $0<h<s$ and write

$$
0<P\left(s, x_{0}, \Gamma\right)=\int_{D\left(A^{\frac{1}{4}}\right)} P\left(t-h, x_{0}, d y\right) P(h, y, \Gamma),
$$

so there exists $y_{0} \in D\left(A^{\frac{1}{4}}\right)$ such that $P\left(h, y_{0}, \Gamma\right)>0$. We can now repeat the first step to show that $P(s, x, \Gamma)>0$ also in this case, which completes the proof.

Lemma 4.1. Assume (A3). Let $P(t, x,),. t>0$ and $x \in H$, be the transition probabilities associated to the Navier-Stokes equation (5). If they are equivalent on $D\left(A^{\frac{1}{4}}\right)$, for all $t>0$ and $x \in D\left(A^{\frac{1}{4}}\right)$, then they are equivalent on $H$, for all $t>0$ and $x \in H$.

Proof. Assume $P\left(t, x_{0}, \Gamma\right)>0$ holds for some $t>0, x_{0} \in H, \Gamma \in \mathscr{B}(H)$. We have to prove that $P(s, x, \Gamma)>0$ for every $s>0$ and $x \in H$. Take $0<h<\min (s, t)$. We have that

$$
\begin{aligned}
0<P\left(t, x_{0}, \Gamma\right) & =\int_{H} P\left(t-h, x_{0}, d y\right) P(h, y, \Gamma) \\
& =\int_{D\left(A^{\frac{1}{4}}\right)} P\left(t-h, x_{0}, d y\right) P(h, y, \Gamma) .
\end{aligned}
$$

The last equality holds since, for every $r>0$, the distribution of $u\left(r ; x_{0}\right)$ is concentrated on $D\left(A^{\frac{1}{4}}\right)$, by Theorem 2.1 (iv), therefore

$$
P\left(t-h, x_{0}, D\left(A^{\frac{1}{4}}\right)\right)=1 .
$$

Therefore, there exists an $y_{0} \in D\left(A^{\frac{1}{4}}\right)$ such that

$$
P\left(h, y_{0}, \Gamma\right)=P\left(h, y_{0}, \Gamma \cap D\left(A^{\frac{1}{4}}\right)\right)>0 .
$$

By the equivalence of the transition probabilities on $D\left(A^{\frac{1}{4}}\right)$, we get that $P(h, y, \Gamma \cap$ $\left.D\left(A^{\frac{1}{4}}\right)\right)>0$ holds for all $y \in D\left(A^{\frac{1}{4}}\right)$. Therefore

$$
\begin{aligned}
P(s, x, \Gamma) & =P\left(s, x, \Gamma \cap D\left(A^{\frac{1}{4}}\right)\right) \\
& =\int_{D\left(A^{\frac{1}{4}}\right)} P(s-h, x, d y) P\left(h, y, \Gamma \cap D\left(A^{\frac{1}{4}}\right)\right)>0 .
\end{aligned}
$$

The proof is complete. 
Collecting the previous theorem and lemma, and recalling Doob's Theorem 3.2, we see that, in order to prove the main Theorem 3.1, we have to check conditions (I) and (SF). This will be the object of the next two sections.

\section{Irreducibility (Condition (I))}

In this section we work under the following assumption: ing

(A4) $G: H \rightarrow H$ is a bounded linear operator, with dense range $\mathscr{R}(G)$, satisfy-

$$
\mathscr{R}(G) \subset D\left(A^{\frac{3}{8}+\varepsilon}\right)
$$

for some $\varepsilon>0$.

Of course $(\mathrm{A} 1) \Rightarrow(\mathrm{A} 4) \Rightarrow(\mathrm{A} 3) \Rightarrow(\mathrm{A} 2)$. Some of the following partial results will be stated assuming only (A2) or (A3).

Our aim is to prove the following result.

Proposition 5.1. Assume that (A4) holds true. Then the irreducibility property (I) is satisfied.

In order to prove this proposition we need several technical lemmas.

First, note that the assumption $\mathscr{R}(G) \subset D\left(A^{\frac{3}{8}+\varepsilon}\right)$ implies that $A^{\frac{3}{8}+\varepsilon} G$ is a bounded operator in $H$ (by the closed graph theorem). Since $A^{-\frac{1}{2}-\frac{\varepsilon}{2}}$ is HilbertSchmidt in $H$, we obtain that

$$
A^{-\frac{1}{8}+\frac{\varepsilon}{2}} G=A^{-\frac{1}{2}-\frac{\varepsilon}{2}} A^{\frac{3}{8}+\varepsilon} G
$$

is Hilbert-Schmidt in $H$. This fact will be used below.

Let $z$ be the Ornstein-Uhlenbeck process defined in Sect. 2.1. It is given by the formula

$$
z(t)=\int_{0}^{t} e^{-(t-s) A} G d w(s) .
$$

By the condition (A3) (which is part of assumption (A4)), $z$ can be represented as the stochastic convolution integral

$$
z(t)=\int_{0}^{t} e^{-(t-s) A} A^{\frac{1}{8}-\frac{\varepsilon}{2}} d \tilde{w}(s),
$$

where $\tilde{w}(s)$ is an $H$-valued Wiener process with nuclear incremental covariance operator

$$
Q=A^{-\frac{1}{8}+\frac{\varepsilon}{2}} G G^{*} A^{-\frac{1}{8}+\frac{\varepsilon}{2}} .
$$

Thus, by Theorem 5.16 of [9], we obtain the following lemma. For a Banach space $Y$, set

$$
C_{0}([0, T] ; Y)=\{h \in C([0, T] ; Y) ; h(0)=0\} .
$$

Lemma 5.1. Under the assumption (A3) (hence under (A4)), we have that

$$
P\left\{z(., \omega) \in C_{0}\left([0, T] ; D\left(A^{\frac{3}{8}}\right)\right)\right\}=1 .
$$


In particular,

$$
P\left\{z(., \omega) \in L^{4}\left(0, T ; D\left(A^{\frac{3}{8}}\right)\right)\right\}=P\left\{z(., \omega) \in C_{0}\left([0, T] ; D\left(A^{\frac{1}{4}}\right)\right)\right\}=1 .
$$

Furthermore, since the function $g(t)=\|S(t)\|_{L\left(H, D\left(A^{\frac{3}{8}}\right)\right)}$ belongs to $L^{2}(0, T)$ by the analyticity of the semigroup $e^{-t A}$, and since the range $\mathscr{R}(G)$ is dense in $H$, we can use Proposition 2.13 in [16] to obtain the following lemma:

Lemma 5.2. Under the assumption (A4), the Gaussian measure induced by $z$ in the space $C_{0}\left([0, T] ; D\left(A^{\frac{3}{8}}\right)\right)$ is full, that is

$$
P\left\{z(., \omega) \in U_{0}\right\}>0
$$

for every nonempty open set $U_{0} \subset C_{0}\left([0, T] ; D\left(A^{\frac{3}{8}}\right)\right)$. Consequently,

$$
P\left\{z(., \omega) \in U_{1}\right\}>0, \quad P\left\{z(., \omega) \in U_{2}\right\}>0
$$

hold true for all sets $U_{1} \subset L^{4}\left(0, T ; D\left(A^{\frac{3}{8}}\right)\right)$ and $U_{2} \subset C_{0}\left([0, T] ; D\left(A^{\frac{1}{4}}\right)\right), U_{1}$ and $U_{2}$ nonempty and open in the respective topologies.

For the sake of brevity, set

$$
K_{\frac{1}{4}}=L^{4}\left(0, T ; D\left(A^{\frac{1}{4}}\right)\right), \quad K_{\frac{3}{8}}=L^{4}\left(0, T ; D\left(A^{\frac{3}{8}}\right)\right),
$$

and for $x \in H$ and $\psi:[0, T] \rightarrow H$ set

$$
u(t, x, \psi)=v(t, x, \psi)+\psi(t),
$$

where $v(t, x, \psi)$ stands for the solution, when it exists, of the equation

$$
\left\{\begin{array}{l}
\frac{d v}{d t}+A v+B(v+\psi, v+\psi)=f, \quad t \in[0, T] . \\
v(0)=x .
\end{array}\right.
$$

As it is sketched in the appendix (see [11] or [22] for more details), this equation has a unique solution

$$
v \in C([0, T] ; H) \cap L^{2}(0, T ; V)
$$

for all $x \in H$ and $\psi \in K_{\frac{1}{4}}$, under the assumption (A2). Moreover, the process $u(t)=$ $v(t, x, z)+z(t)$, where $z$ is the Ornstein-Uhlenbeck process, is the solution of the stochastic Navier-Stokes equation (5), described in part ii) of Theorem 2.1. If in addition $x \in D\left(A^{\frac{1}{4}}\right)$ and $\psi \in K_{\frac{3}{8}}$, and (A3) is assumed, then $v \in C\left([0, T] ; D\left(A^{\frac{1}{4}}\right)\right)$.

Lemma 5.3. (a) Under the assumption (A2), for every $x \in H$, the mapping

$$
\Phi: K_{\frac{1}{4}} \cap C_{0}([0, T] ; H) \rightarrow C([0, T] ; H)
$$

defined as $\Phi(\psi)=u(., x, \psi)$, is continuous.

(b) Under the assumption (A3), for every $x \in D\left(A^{\frac{1}{4}}\right)$, the restriction of $\Phi$ :

$$
\Phi: K_{\frac{3}{8}} \cap C_{0}\left([0, T] ; D\left(A^{\frac{1}{4}}\right)\right) \rightarrow C\left([0, T] ; D\left(A^{\frac{1}{4}}\right)\right)
$$

maps bounded sets in bounded sets. 
(c) Under the assumption (A3), for every $T>0$ and every $x, y \in D\left(A^{\frac{1}{4}}\right)$, there exists $\bar{z} \in K_{\frac{3}{8}} \cap C_{0}\left([0, T] ; D\left(A^{\frac{1}{4}}\right)\right)$ such that $u(T, x, \bar{z})=y$.

Proof. Part (a) is proved in the Appendix, Remark A.2, while (b) in Remark A.1. Thus we have only to prove (c).

Given $x, y \in D\left(A^{\frac{1}{4}}\right)$ and $T>0$, choose any $0<t_{0}<t_{1}<T$. Define $\bar{u}$ as:

$$
\begin{aligned}
& \bar{u}(t)=e^{-t A} x, \quad t \in\left[0, t_{0}\right], \\
& \bar{u}(t)=e^{-(T-t) A} y, \quad t \in\left[t_{1}, T\right], \\
& \bar{u}(t)=\bar{u}\left(t_{0}\right)+\frac{t-t_{0}}{t_{1}-t_{0}}\left(\bar{u}\left(t_{1}\right)-\bar{u}\left(t_{0}\right)\right), \quad t \in\left(t_{0}, t_{1}\right) .
\end{aligned}
$$

We have $\bar{u} \in C\left([0, T] ; D\left(A^{\frac{1}{4}}\right)\right)$. Moreover, by the analyticity of the semigroup,

$$
\begin{aligned}
\int_{0}^{t_{0}}\|\bar{u}(s)\|^{2} d s & \leqq C_{1} \int_{0}^{t_{0}}\left|A^{\frac{1}{4}} e^{-s A}\right|_{L(H)}^{2} d s \\
& \leqq C_{2} \int_{0}^{t_{0}} \frac{1}{s^{\frac{1}{2}}} d s<\infty,
\end{aligned}
$$

and similarly on $\left[t_{1}, T\right]$, while on $\left[t_{0}, t_{1}\right]$ the function $\bar{u}$ is even continuous with values in $D\left(A^{N}\right)$ for each $N>0$. Therefore $\bar{u} \in L^{2}(0, T ; V)$. Along with the previous property of $\bar{u}$, and a classical interpolation inequality, this implies

$$
\int_{0}^{T}\left|A^{\frac{3}{8}} \bar{u}(s)\right|^{4} d s \leqq C_{3} \int_{0}^{T}\left|A^{\frac{1}{4}} \bar{u}(s)\right|^{2}\left|A^{\frac{1}{2}} \bar{u}(s)\right|^{2} d s<\infty .
$$

Define now $\bar{v}$ as the solution of the equation

$$
\left\{\begin{array}{l}
\frac{d \bar{v}}{d t}+A \bar{v}=-B(\bar{u}, \bar{u})+f, \quad t \in[0, T] \\
\bar{v}(0)=x
\end{array}\right.
$$

It is well known that if $x \in D\left(A^{\frac{1}{4}}\right)$ and the right-hand side is in $L^{2}\left(0, T ; D\left(A^{\frac{1}{4}}\right)^{\prime}\right)$, then

$$
\bar{v} \in C\left([0, T] ; D\left(A^{\frac{1}{4}}\right)\right) \cap L^{2}\left(0, T ; D\left(A^{\frac{3}{4}}\right)\right)
$$

(cf. [22]). Note that, for all $\xi \in D\left(A^{\frac{1}{4}}\right)$ with norm 1 ,

$$
|\langle B(\bar{u}, \bar{u}), \xi\rangle| \leqq C_{4}\left|A^{\frac{1}{4}} \bar{u}\right|\|\bar{u}\|
$$

so that, by (9), $|B(\bar{u}, \bar{u})|_{D\left(A^{\frac{1}{4}}\right)^{\prime}}$ is square integrable. Since $x \in D\left(A^{\frac{1}{4}}\right)$, we have (12), so that, by (10) (since $\left.D\left(A^{\frac{3}{4}}\right) \subset V\right)$, we finally obtain $\bar{v} \in L^{4}\left(0, T ; D\left(A^{\frac{3}{4}}\right)\right.$ ).

If we now set $\bar{z}=\bar{u}-\bar{v}$, it is straightforward to check that it satisfies all the requirements of the lemma, part (c). This completes the proof.

Proof of Proposition 5.1. Let T, $x$ and $y$ be given as in the proposition. Let $\bar{z}$ be given by part (c) of the last lemma. By part (a), there exists $\delta_{0}>0$ such that

$$
|z-\bar{z}|_{\frac{1}{4}}+|z-\bar{z}|_{C_{0}([0, T] ; H)}<\delta_{0}
$$


implies

$$
|u(., x, z)-u(., x, \bar{z})|_{C([0, T] ; H)}<1 .
$$

Find $\delta_{1}>0$ such that

$$
|z-\bar{z}|_{K_{\frac{3}{8}}}+|z-\bar{z}|_{C_{0}\left([0, T] ; D\left(A^{\frac{1}{4}}\right)\right)}<\delta_{1}
$$

implies

$$
|z-\bar{z}|_{\frac{1}{4}}+|z-\bar{z}|_{C_{0}([0, T] ; H)}<\delta_{0},
$$

for $z \in K_{\frac{3}{8}} \cap C_{0}\left([0, T] ; D\left(A^{\frac{1}{4}}\right)\right)$. By (b) we have that

$$
M=\sup \left\{|u(., x, z)|_{C\left([0, T] ; D\left(A^{\frac{1}{4}}\right)\right)} ;|z-\bar{z}|_{K_{\frac{3}{8}}}+|z-\bar{z}|_{C_{0}\left([0, T] ; D\left(A^{\frac{1}{4}}\right)\right)}<\delta_{1}\right\}<\infty .
$$

Now take any $0<\varepsilon<1$ and find $\delta>0, \delta<\delta_{0}$, such that

$$
|u(., x, z)-u(., x, \bar{z})|_{C([0, T] ; H)}<\varepsilon
$$

holds for $z$ satisfying

$$
|z-\bar{z}|_{K_{\frac{1}{4}}}+|z-\bar{z}|_{C_{0}([0, T] ; H)}<\delta,
$$

and then take $\Delta>0, \Delta<\delta_{1}$, such that

$$
z \in U_{\Delta}=\left\{z \in K_{\frac{3}{8}} \cap C_{0}\left([0, T] ; D\left(A^{\frac{1}{4}}\right)\right) ;|z-\bar{z}|_{K_{\frac{3}{8}}}+|z-\bar{z}|_{C_{0}\left([0, T] ; D\left(A^{\frac{1}{4}}\right)\right)}<\Delta\right\}
$$

implies

$$
|z-\bar{z}|_{K_{\frac{1}{4}}}+|z-\bar{z}|_{C_{0}([0, T] ; H)}<\delta .
$$

For $z \in U_{\Delta}$, we have that

$$
|u(., x, z)|_{C\left([0, T] ; D\left(A^{\frac{1}{4}}\right)\right)} \leqq M
$$

and

$$
|u(T, x, z)-y|<\varepsilon .
$$

Recall now that the solution $u$ of the stochastic Navier-Stokes equation is equal to $\Phi(z), z$ being the Ornstein-Uhlenbeck process. Then it remains to show that

$$
P\left\{z(., \omega) \in U_{\Delta}\right\}>0,
$$

which follows from Lemma 5.2. The proof of the proposition is complete.

\section{Strong Feller Property (Condition (SF))}

In this section we prove that the condition (SF) of Sect. 4 holds true, which will complete the proof of Theorem 3.1. To this end we adopt the following strategy: at first we prove the classical strong Feller property on $H$ for Eq. (13) below which differs from the Navier-Stokes equation by a regularizing multiplicative term $\Theta_{R}$ 
(we are not able to prove the strong Feller property for the original equation directly). Then we prove the condition (SF) for the original equation by the limit passage for $R \rightarrow \infty$.

In order to prove the strong Feller property for Eq. (13) with $R>0$ fixed we use the finite dimensional Galerkin approximation. For the crucial estimation of the derivative of the transition probability functions in Lemma 6.2 we use an interesting formula involving directional derivatives of the stochastic equations, which can be derived from the backward Kolmogorov equation and the Ito formula (cf. [7, 19] for details).

6.1. Modified Navier-Stokes Equation. Fix $R>0$ and let $\Theta_{R}$ be a $C_{\infty}$ function equal to 1 in $[-R, R]$ and to 0 outside $[-R-1, R+1]$. Consider the equation

$$
\left\{\begin{array}{l}
d u^{(R)}(t)+A u^{(R)}(t) d t+\Theta_{R}\left(\left|u^{(R)}\right|_{D\left(A^{\frac{1}{4}}\right)}^{2}\right) B\left(u^{(R)}(t), u^{(R)}(t)\right) d t \\
=f d t+G d w(t), \quad t \in[0, T] \\
u^{R}(0)=u_{0}
\end{array}\right.
$$

Lemma 6.1. Assume condition (A3). Then, for all $u_{0} \in D\left(A^{\frac{1}{4}}\right)$, there exists a unique generalized solution of Eq. (13) (in the sense of Definition 2.1, modified by the term with $\Theta_{R}$ ) satisfying

$$
u(., \omega) \in C\left([0, T] ; D\left(A^{\frac{1}{4}}\right)\right) \cap L^{2}\left(0, T ; D\left(A^{\frac{3}{8}}\right)\right)
$$

and

$$
u(., \omega)-z(., \omega) \in L^{2}(0, T ; V)
$$

for P-a.e. $\omega \in \Omega$, and it generates a Markov process, with the Feller property, in $D\left(A^{\frac{1}{4}}\right)$.

The proof is the same as that of Theorem 2.1, and it is based on the estimates 1 and 2 of the appendix and the limit argument recalled in Remark A.1, which are completely similar for the original Eq. (5) and for the modified Eq. (13). Denote by $P_{R}=P_{R}(t, x,$.$) the transition probabilities corresponding to (13)$, and set

$$
P_{t}^{(R)} \phi(x)=\int \phi(y) P_{R}(t, x, d y)
$$

for $x \in D\left(A^{\frac{1}{4}}\right)$ and $\phi \in C_{b}\left(D\left(A^{\frac{1}{4}}\right)\right)$ (the space $C_{b}\left(D\left(A^{\frac{1}{4}}\right)\right)$ includes the restriction of the functions $\left.\phi \in C_{b}(H)\right)$.

Proposition 6.1. Assume (A1). Then for every $R>0, t>0$, there exists a constant $L=L(t, R)>0$ such that

$$
\left|P_{t}^{(R)} \phi(x)-P_{t}^{(R)} \phi(y)\right| \leqq L|x-y|
$$

holds for all $x, y \in D\left(A^{\frac{1}{4}}\right)$, and all $\phi \in C_{b}\left(D\left(A^{\frac{1}{4}}\right)\right)$ with $|\phi|_{C_{b}\left(D\left(A^{\frac{1}{4}}\right)\right)} \leqq 1$.

The proof will follow from a corresponding result for the following Galerkin approximations of Eq. (13). Let $P_{n}$ be the orthogonal projection in $H$ defined as $P_{n} x=\sum_{j=1}^{n}\left\langle x, e_{j}\right\rangle e_{j}, x \in H$. Clearly, $H_{n}:=P_{n} H \subset D\left(A^{N}\right)$ for every $N>0$. 
Consider the equation in $H_{n}$ :

$$
\left\{\begin{array}{l}
d u_{n}^{(R)}(t)+A u_{n}^{(R)}(t) d t+\Theta_{R}\left(\left|u_{n}^{(R)}\right|_{D\left(A^{\frac{1}{4}}\right)}^{2}\right) P_{n} B\left(u_{n}^{(R)}(t), u_{n}^{(R)}(t)\right) d t \\
=P_{n} f d t+P_{n} G d w(t), \quad t \in[0, T] \\
u_{n}^{(R)}(0)=P_{n} u_{0}
\end{array}\right.
$$

This is a finite dimensional equation with globally Lipschitz nonlinear functions, so it has a unique progressively measurable solution with $P$-a.e. trajectory $u_{n}^{(R)}(., \omega) \in$ $C\left([0, T] ; H_{n}\right)$, generating a Markov process in $H_{n}$ with associated semigroup $P_{n, t}^{(R)}$ defined as

$$
P_{n, t}^{(R)} \psi(x)=E \psi\left(u_{n}^{(R)}(t ; x)\right)
$$

for all $x \in H_{n}$ and $\psi \in C_{b}\left(H_{n}\right)$.

Lemma 6.2. Assume (A1). Then for every $R>0, t>0$, there exists a constant $L=L(t, R)>0$ such that

$$
\left|P_{n, t}^{(R)} \phi(x)-P_{n, t}^{(R)} \phi(y)\right| \leqq L|x-y|
$$

holds for all $n \in \mathbf{N}, x, y \in H_{n}$, and all $\phi \in C_{b}\left(H_{n}\right)$ with $|\phi|_{H_{n}} \leqq 1$.

Proof. The following remarkable formula holds true for the differential in $x$ of $P_{n, t}^{(R)} \phi$ (cf. $[7,19])$ :

$$
D_{x} P_{n, t}^{(R)} \phi(x) \cdot h=\frac{1}{t} E\left(\psi\left(u_{n}^{(R)}(t ; x)\right) \int_{0}^{t}\left\langle\left(P_{n} G G^{*} P_{n}\right)^{-\frac{1}{2}} D_{x} u_{n}^{(R)}(s ; x) \cdot h, d \beta_{n}(s)\right\rangle\right)
$$

for all $h \in H_{n}$, where $\beta_{n}$ is a $n$-dimensional standard Wiener process. We have used the fact that $P_{n} G w(t)$ is a $n$-dimensional Wiener process with incremental covariance $P_{n} G G^{*} P_{n}$. Therefore,

$$
\left|D_{x} P_{n, t}^{(R)} \phi(x) \cdot h\right| \leqq \frac{1}{t} E\left(\int_{0}^{t}\left|\left(P_{n} G G^{*} P_{n}\right)^{-\frac{1}{2}} D_{x} u_{n}^{(R)}(s ; x) \cdot h\right|^{2} d s\right)^{\frac{1}{2}} .
$$

We use now the fact, proved below, that there exists a constant $C>0$, independent of $n$, such that

$$
\left|\left(P_{n} G G^{*} P_{n}\right)^{-\frac{1}{2}} y\right|^{2} \leqq C\|y\|^{2} \quad \forall y \in H_{n}
$$

It follows that

$$
\begin{aligned}
\left|D_{x} P_{n, t}^{(R)} \phi(x) \cdot h\right| & \leqq \frac{1}{t} C E\left(\int_{0}^{t}\left\|D_{x} u_{n}^{(R)}(s ; x) \cdot h\right\|^{2} d s\right)^{\frac{1}{2}} \\
& \leqq \frac{1}{t} C(R)|h|
\end{aligned}
$$

by the estimate 4 of the appendix (note that $C(R)$ is independent of $x \in H_{n}$ and $n \in \mathbf{N}$. Indeed, $u_{n}^{(R)}(t ; x)$ is given by $v_{n}(t ; x)+P_{n} z(t)$, where $z$ is the Ornstein- 
Uhlenbeck process, and $v_{n}$ is the solution of Eq. (16) with $\psi=z$ ). Therefore,

$$
\begin{aligned}
\left|P_{n, t}^{(R)} \phi(x)-P_{n, t}^{(R)} \phi(y)\right| & \leqq \sup _{|h| \leqq 1, k \in H_{n}}\left|D_{x} P_{n, t}^{(R)} \phi(k) \cdot h\right||x-y| \\
& \leqq \frac{1}{t} C(R)|x-y|
\end{aligned}
$$

We have to prove (15). For all $x \in H_{n}$,

$$
\begin{aligned}
\left\langle A^{\frac{1}{2}} P_{n} G G^{*} P_{n} A^{\frac{1}{2}} x, x\right\rangle & =\left\langle A^{\frac{1}{2}} G G^{*} A^{\frac{1}{2}} x, x\right\rangle \\
& \geqq \frac{1}{C}|x|^{2}
\end{aligned}
$$

for some constant $C>0$, since, by the assumption $D\left(A^{\frac{1}{2}}\right) \subset \mathscr{R}(G)$,

$$
\left(A^{\frac{1}{2}} G G^{*} A^{\frac{1}{2}}\right)^{-1}=\left(G^{-1} A^{-\frac{1}{2}}\right)^{*} G^{-1} A^{-\frac{1}{2}}
$$

is bounded in $H$. Indeed,

$$
\left(G^{-1} A^{-\frac{1}{2}}\right)^{*} G^{-1} A^{-\frac{1}{2}} A^{\frac{1}{2}} G G^{*} A^{\frac{1}{2}}=\left(G^{-1} A^{-\frac{1}{2}}\right)^{*} G^{*} A^{-\frac{1}{2}}
$$

and, for every $x, y \in V$,

$$
\begin{aligned}
\left\langle\left(G^{-1} A^{-\frac{1}{2}}\right)^{*} G^{*} A^{\frac{1}{2}} x, y\right\rangle & =\left\langle G^{*} A^{\frac{1}{2}} x, G^{-1} A^{-\frac{1}{2}} y\right\rangle \\
& =\left\langle A^{\frac{1}{2}} x, G G^{-1} A^{-\frac{1}{2}} y\right\rangle \\
& =\left\langle A^{\frac{1}{2}} x, A^{-\frac{1}{2}} y\right\rangle \\
& =\left\langle x, A^{\frac{1}{2}} A^{-\frac{1}{2}} y\right\rangle \\
& =\langle x, y\rangle .
\end{aligned}
$$

Therefore,

$$
\left\langle\left(A^{\frac{1}{2}} P_{n} G G^{*} P_{n} A^{\frac{1}{2}}\right)^{-1} y, y\right\rangle \leqq C|y|^{2}
$$

for all $y \in H_{n}$, so that

$$
\begin{aligned}
\left|\left(P_{n} G G^{*} P_{n}\right)^{-\frac{1}{2}} y\right|^{2} & =\left\langle\left(P_{n} G G^{*} P_{n}\right)^{-1} y, y\right\rangle \\
& =\left\langle\left(A^{\frac{1}{2}} P_{n} G G^{*} P_{n} A^{\frac{1}{2}}\right)^{-1} A^{\frac{1}{2}} y, A^{\frac{1}{2}} y\right\rangle \\
& =\left|\left(A^{\frac{1}{2}} P_{n} G G^{*} P_{n} A^{\frac{1}{2}}\right)^{-\frac{1}{2}} A^{\frac{1}{2}} y\right|^{2} \leqq C\|y\|^{2} .
\end{aligned}
$$

The proof of the lemma is complete.

Proof of Proposition 6.1. Let $x \in D\left(A^{\frac{1}{4}}\right)$ and $\psi \in C_{b}\left(D\left(A^{\frac{1}{4}}\right)\right)$ be given (note that $\left.\psi \in C_{b}\left(H_{n}\right)\right)$. From the appendix, Remark A.1, we know that $u_{n}^{(R)}(. ; x)$ converges to $u^{(R)}(. ; x)$ strongly in $L^{2}\left(0, T ; D\left(A^{\frac{1}{4}}\right)\right), P$-a.s. Fix $\omega \in \Omega$ such that this property holds. For this $\omega$, by a simple argument on subsequences that converge a.s. in $t$, we see that $\phi\left(u_{n}^{(R)}(. ; x)\right)$ converges to $\phi\left(u^{(R)}(. ; x)\right)$ in $L^{1}(0, T)$ (we use the continuity 
and boundedness of $\phi$ on $\left.D\left(A^{\frac{1}{4}}\right)\right)$. Again by the boundedness of $\phi$ and Lebesgue dominated convergence theorem, we have

$$
E \int_{0}^{T}\left|\phi\left(u_{n}^{(R)}(t ; x)\right)-\phi\left(u^{(R)}(t ; x)\right)\right| d t \rightarrow 0,
$$

which implies that for some subsequence $n_{k}$

$$
E \phi\left(u_{n_{k}}^{(R)}(t ; x)\right) \rightarrow E \phi\left(u^{(R)}(t ; x)\right)
$$

for a.e. $t \in[0, T]$.

Take now $x, y \in D\left(A^{\frac{1}{4}}\right)$. By the previous argument, we can find a subsequence $n_{k}$ such that the previous almost sure convergence in $t \in[0, T]$ holds true both $x$ and $y$. Thus, from Lemma 6.2, we have

$$
\left.\mid P_{t}^{(R)} \phi(x)-P_{t}^{(R)} \phi(y)\right)|\leqq L| x-y \mid
$$

for a.e. $t \in[0, T]$. It is now standard to show that $P_{t}^{(R)} \phi(x)$ is continuous in $t$, for all $x \in D\left(A^{\frac{1}{4}}\right)$, since the Markov process $u^{(R)}(t ; x)$ has continuous trajectories with values in $D\left(A^{\frac{1}{4}}\right)$. It follows that the previous inequality holds for all $t \in[0, T]$, completing the proof of the proposition.

Lemma 6.3. Under the assumption (A3), we have that for every $t>0, M>0$,

$$
\left\|P_{R}(t, x, .)-P(t, x, .)\right\|_{v} \rightarrow 0
$$

as $R \rightarrow \infty$, uniformly with respect to

$$
x \in B_{M}:=\left\{x \in D\left(A^{\frac{1}{4}}\right):\left|A^{\frac{1}{4}} x\right| \leqq M\right\} .
$$

Proof. Let $u(t, x, \psi)$ have the same meaning as in Lemma 5.3. Since we have that

$$
\sup \left\{\left|A^{\frac{1}{4}} u(t, x, \psi)\right|: x \in B_{M}\right\}<\infty
$$

for every $\psi \in C_{0}\left([0, T] ; D\left(A^{\frac{1}{4}}\right)\right)$ (cf. Remark A.1 in the appendix), we obtain

$$
P\left(\sup \left\{\left|A^{\frac{1}{4}} u(t ; x)\right|: x \in B_{M}\right\}>\infty\right) \rightarrow 1
$$

as $R \rightarrow \infty$, where $u(t ; x)$ stands for the solution of the original Eq. (5) satisfying $u(0 ; x)=x$. This completes the proof of Lemma 6.3 , since the trajectories of the processes $u(. ; x)$ and $u^{(R)}(. ; x)$ coincide on $B_{R}$ for $x \in B_{M}$.

Proposition 6.2. Assume (A1). Then (SF) holds true.

Proof. Take $t>0, x_{n}, x \in D\left(A^{\frac{1}{4}}\right),\left|A^{\frac{1}{4}} x_{n}\right| \leqq C$ for a constant $C>0, x_{n} \rightarrow x$ in $H$. For every $R>0$ we have that

$$
\begin{aligned}
\left\|P_{R}\left(t, x_{n}, .\right)-P_{R}(t, x, .)\right\|_{v} & =\sup _{\|\phi\|_{C_{b}(H)} \leqq 1}\left|P_{t}^{(R)} \phi\left(x_{n}\right)\right|-\left|P_{t}^{(R)} \phi(x)\right| \\
& \leqq L\left|x_{n}-x\right| \rightarrow 0
\end{aligned}
$$


as $n \rightarrow \infty$ by Proposition 6.1. Furthermore,

$$
\left\|P_{R}\left(t, x_{n}, .\right)-P\left(t, x_{n}, .\right)\right\|_{v}+\left\|P_{R}(t, x, .)-P(t, x, .)\right\|_{v} \rightarrow 0
$$

as $R \rightarrow \infty$, uniformly with respect to $n \in \mathbf{N}$, by Lemma 6.3 . Therefore,

$$
\begin{aligned}
\left\|P\left(t, x_{n}, .\right)-P(t, x, .)\right\|_{v} \leqq & \left\|P\left(t, x_{n}, .\right)-P_{R}\left(t, x_{n}, .\right)\right\|_{v}+\left\|P_{R}\left(t, x_{n}, .\right)-P_{R}(t, x, .)\right\|_{v} \\
& +\left\|P_{R}(t, x, .)-P(t, x, .)\right\|_{v} \rightarrow 0
\end{aligned}
$$

$n \rightarrow \infty$, and the proof of (SF) is completed.

\section{Appendix: A Priori Estimates}

Let $\Theta_{R}, R>0$, be a $C^{\infty}$ function as in Sect. 6. We allow also the value $R=\infty$, in which case we set $\Theta_{R}$ identically equal to 1 .

Consider the equation

$$
\left\{\begin{array}{l}
\frac{d \iota_{n}}{d t}+A v_{n}+\Theta_{R}\left(\left|v_{n}+P_{n} \psi\right|_{D\left(A^{\frac{1}{4}}\right)}^{2}\right) P_{n} B\left(v_{n}+P_{n} \psi, v_{n}+P_{n} \psi\right)=P_{n} f, \quad t \in[0, T] \\
v_{n}(0)=P_{n} x .
\end{array}\right.
$$

Here $\psi \in K_{\frac{1}{4}}$ (see Sect. 5) is a given function, $x \in H$. In this appendix we prove some a priori estimates on $v_{n}$.

First we recall the following result from [11]:

Lemma 7.1. For all $\varepsilon>0$ there exists a constant $C(\varepsilon)>0$ such that: for all $v \in V$ and $z \in D\left(A^{\frac{1}{4}}\right)$,

$$
|\langle B(v+z, v+z), v\rangle| \leqq \varepsilon\|v\|^{2}+C(\varepsilon)\left|A^{\frac{1}{4}} z\right|^{4}|v|^{2}+C(\varepsilon)\left|A^{\frac{1}{4}} z\right|^{4}
$$

for all $v \in D\left(A^{\frac{3}{4}}\right), z \in D\left(A^{\frac{3}{8}}\right)$

$$
\left\langle A^{\frac{1}{2}} v, B(v+z, v+z)\right\rangle \leqq \varepsilon\left|A^{\frac{3}{4}} v\right|^{2}+C(\varepsilon)\left\{|v|^{2}\left|A^{\frac{1}{2}} v\right|^{2}\left|A^{\frac{1}{4}} v\right|^{2}+\left|A^{\frac{3}{8}} z\right|^{4}\right\}
$$

In the following estimates, we shall denote by $C$ a generic positive constant independent of $n, \psi, x, R, f$.

Estimate 1. We prove the following a priori bound in $H$ : there exists a constant $C\left(|x|,|\psi|_{K_{\frac{1}{4}}}, T,|f|\right)$, depending only on the indicated arguments, such that

$$
\left|v_{n}\right|_{C([0, T] ; H)}+\left|v_{n}\right|_{L^{2}(0, T ; V)} \leqq C\left(|x|,|\psi|_{K_{\frac{1}{4}}}, T,|f|\right)
$$

for every $R, n, x \in H, \psi \in K_{\frac{1}{4}}, T, f \in H$. The space $K_{\frac{1}{4}}$ is defined in Sect. 5 . 
We have

$$
\begin{aligned}
\frac{1}{2} \frac{d}{d t}\left|v_{n}\right|^{2}+\left\|v_{n}\right\|^{2} & =-\Theta_{R}\left(\left|v_{n}+P_{n} \psi\right|_{D\left(A^{\frac{1}{4}}\right)}^{2}\right)\left\langle B\left(v_{n}+P_{n} \psi, v_{n}+P_{n} \psi\right), v_{n}\right\rangle+\left\langle f, v_{n}\right\rangle \\
& \leqq \varepsilon\left\|v_{n}\right\|^{2}+C(\varepsilon)\left|A^{\frac{1}{4}} \psi\right|^{4}\left|v_{n}\right|^{2}+C(\varepsilon)\left|A^{\frac{1}{4}} \psi\right|^{4}+C(\varepsilon)|f|_{V^{\prime}}^{2}+\varepsilon\|v\|^{2}
\end{aligned}
$$

Here we have used the first part of the previous lemma, and also the fact that $P_{n}$ commutes with $A$ and is equibounded by 1 in $L(H)$. Moreover, $\varepsilon$ is an arbitrary positive number and $C(\varepsilon)$ is a positive constant. Taking $\varepsilon$ sufficiently small (and denoting the corresponding constant by $C$ ) we have

$$
\frac{1}{2} \frac{d}{d t}\left|v_{n}\right|^{2}+\frac{1}{2}\left\|v_{n}\right\|^{2} \leqq C\left|A^{\frac{1}{4}} \psi\right|^{4}\left|v_{n}\right|^{2}+C\left|A^{\frac{1}{4}} \psi\right|^{4}+C|f|_{V^{\prime}}^{2}
$$

Therefore, for all $t \in[0, T]$ (renaming $C$ )

$$
\left|v_{n}(t)\right|^{2} \leqq e^{\int_{0}^{t} C\left|A^{\frac{1}{4}} \psi(s)\right|^{4} d s}|x|^{2}+\int_{0}^{t} e^{\int_{\sigma}^{t} C\left|A^{\frac{1}{4}} \psi(s)\right|^{4} d s} C\left(\left|A^{\frac{1}{4}} \psi(\sigma)\right|^{4}+|f|_{V^{\prime}}^{2}\right) d \sigma .
$$

Using this result and the previous inequality, we also obtain (renaming $C$ )

$$
\int_{0}^{T}\left\|v_{n}(\sigma)\right\|^{2} d \sigma \leqq|x|^{2}+\int_{0}^{T} C\left(\left|A^{\frac{1}{4}} \psi\right|^{4}\left|v_{n}\right|^{2}+\left|A^{\frac{1}{4}} \psi\right|^{4}+|f|_{V^{\prime}}^{2}\right) d \sigma
$$

This proves (19).

Estimate 2. We prove the following a priori bound in $D\left(A^{\frac{1}{4}}\right)$ : there exists a constant $C\left(\left|A^{\frac{1}{4}} x\right|,|\psi|_{K_{\frac{3}{8}}}, T,|f|\right)$, depending only on the indicated arguments, such that

$$
\left|v_{n}\right|_{C\left([0, T] ; D\left(A^{\frac{1}{4}}\right)\right)}+\left|v_{n}\right|_{L^{2}\left(0, T ; D\left(A^{\frac{3}{4}}\right)\right)} \leqq C\left(\left|A^{\frac{1}{4}} x\right|,|\psi|_{K_{\frac{3}{8}}}, T,|f|\right)
$$

for every $R, n, x \in D\left(A^{\frac{1}{4}}\right), \psi \in K_{\frac{3}{8}}, T, f \in H$. The space $K_{\frac{3}{8}}$ is defined in Sect. 5 .

Assume that $x \in D\left(A^{\frac{1}{4}}\right), \psi \in K_{\frac{3}{8}}$. We have

$$
\begin{aligned}
\frac{1}{2} \frac{d}{d t}\left|A^{\frac{1}{4}} v_{n}\right|^{2}+\left|A^{\frac{3}{4}} v_{n}\right|^{2}= & -\Theta_{R}\left(\left|v_{n}+P_{n} \psi\right|_{D\left(A^{\frac{1}{4}}\right)}^{2}\right) \\
& \times\left\langle A^{\frac{1}{4}} B\left(v_{n}+P_{n} \psi, v_{n}+P_{n} \psi\right), A^{\frac{1}{4}} v_{n}\right\rangle+\left\langle A^{\frac{1}{4}} f, A^{\frac{1}{4}} v_{n}\right\rangle \\
\leqq & \varepsilon\left|A^{\frac{3}{4}} v_{n}\right|^{2}+C(\varepsilon)\left\{\left|v_{n}\right|^{2}\left|A^{\frac{1}{2}} v_{n}\right|^{2}\left|A^{\frac{1}{4}} v_{n}\right|^{2}+\left|A^{\frac{3}{8}} \psi\right|^{4}\right\} \\
& +\varepsilon\left|A^{\frac{3}{4}} v_{n}\right|^{2}+C(\varepsilon)\left|A^{-\frac{1}{4}} f\right|^{2}
\end{aligned}
$$

by the second part of the previous lemma. For a suitable $\varepsilon$ we have

$$
\frac{1}{2} \frac{d}{d t}\left|A^{\frac{1}{4}} v_{n}\right|^{2}+\frac{1}{2}\left|A^{\frac{3}{4}} v_{n}\right|^{2} \leqq\left. C\left|v_{n}\right|^{2} A^{\frac{1}{2}} v_{n}\right|^{2}\left|A^{\frac{1}{4}} v_{n}\right|^{2}+C\left|A^{\frac{3}{8}} \psi\right|^{4}+C|f|^{2} .
$$


This implies, for all $t \in[0, T]$ (renaming $C$ )

$$
\begin{aligned}
\left|A^{\frac{1}{4}} v_{n}(t)\right|^{2} \leqq & e^{\int_{0}^{t} C\left|v_{n}(s)\right|^{2}\left|A^{\frac{1}{2}} v_{n}(s)\right|^{2} d s}\left|A^{\frac{1}{4}} x\right|^{2}+\int_{0}^{t} e^{\int_{\sigma}^{t} C\left|v_{n}(s)\right|^{2}\left|A^{\frac{1}{2}} v_{n}(s)\right|^{2} d s} \\
& \times C\left(\left|A^{\frac{3}{8}} \psi(\sigma)\right|^{4}+|f|^{2}\right) d \sigma .
\end{aligned}
$$

Moreover, we also obtain (renaming $C$ )

$$
\int_{0}^{T}\left|A^{\frac{3}{4}} v_{n}(\sigma)\right|^{2} d \sigma \leqq\left|A^{\frac{1}{4}} x\right|^{2}+\int_{0}^{T} C\left(\left|v_{n}\right|^{2}\left|A^{\frac{1}{2}} v_{n}\right|^{2}\left|A^{\frac{1}{4}} v_{n}\right|^{2}+\left|A^{\frac{3}{8}} \psi\right|^{4}+|f|^{2}\right) d \sigma .
$$

Therefore, using also the bound (19), we have proved (22).

Remark. A.1. It is now standard to show that, given $x \in H$ and $\psi \in K_{\frac{1}{4}}$, there exists a subsequence (in fact the full sequence, a fortiori, by the uniqueness of the limit), still denoted by $v_{n}$, which converges to some $v$, strongly in $L^{2}(0, T ; H)$, weakly in $L^{2}(0, T ; V)$, and weak star in $L^{\infty}(0, T ; H)$. Therefore, since

$$
|v|_{L^{\infty}([0, T] ; H)} \leqq \liminf _{n \rightarrow \infty}\left|v_{n}\right|_{L^{\infty}([0, T] ; H)},
$$

and similarly for $|v|_{L^{2}(0, T ; V)}$, we have that

$$
|v|_{L^{\infty}([0, T] ; H)}+|v|_{L^{2}(0, T ; V)} \leqq C\left(|x|,|\psi|_{K_{\frac{1}{4}}}, T,|f|\right)
$$

for every $R, x \in H, \psi \in K_{\frac{1}{4}}, T, f \in H$. When $R=\infty, v$ satisfies Eq. (8).

If, in addition, $x \in D\left(A^{\frac{1}{4}}\right)$ and $\psi \in K_{\frac{3}{8}}$, then $v_{n}$ also converges strongly in $L^{2}\left(0, T ; D\left(A^{\frac{1}{4}}\right)\right)$, weakly in $L^{2}\left(0, T ; D\left(A^{\frac{3}{4}}\right)\right)$, and weak star in $L^{\infty}\left(0, T ; D\left(A^{\frac{1}{4}}\right)\right)$, to $v$. Therefore,

$$
|v|_{L^{\infty}\left([0, T] ; D\left(A^{\frac{1}{4}}\right)\right)}+|v|_{L^{2}\left(0, T, D\left(A^{\frac{3}{4}}\right)\right)} \leqq C\left(\left|A^{\frac{1}{4}} x\right|,|\psi|_{K_{\frac{3}{8}}}, T,|f|\right)
$$

for every $R, x \in D\left(A^{\frac{1}{4}}\right), \psi \in K_{\frac{3}{8}}, T, f \in H$. Moreover, for each $R, v$ satisfies Eq. (13). Uniqueness of solution to these equations can be proved with computations like those of Estimate 3 below. Also the proof that $v \in C([0, T] ; H)$ in the first case, and $v \in C\left([0, T] ; D\left(A^{\frac{1}{4}}\right)\right)$ in the second one, is classical.

Estimate 3. We compare, only in the case $R=\infty$ for simplicity, two solutions $v_{n}^{1}, v_{n}^{2}$ corresponding to the same initial condition $x \in H$ but to different functions $\psi^{1}, \psi^{2}$ : there exists a constant $C\left(|x|,\left|\psi^{1}\right|_{K_{\frac{1}{4}}},\left|\psi^{2}\right|_{K_{\frac{1}{4}}} T,|f|\right)$, depending only on the indicated arguments, such that

$$
\left|v_{n}^{1}-v_{n}^{2}\right|_{C([0, T] ; H)} \leqq C\left(|x|,\left|\psi^{1}\right|_{K_{\frac{1}{4}}},\left|\psi^{2}\right|_{K_{\frac{1}{4}}} T,|f|\right)\left|\psi^{1}-\psi^{2}\right|_{K_{\frac{1}{4}}}
$$

for every $n, x \in H, \psi^{2}, \psi^{2} \in K_{\frac{1}{4}}, T, f \in H$. 
We have

$$
\left\{\begin{array}{l}
\frac{d v_{n}^{l}}{d t}+A v_{n}^{i}+P_{n} B\left(v_{n}^{l}+P_{n} \psi^{i}, v_{n}^{i}+P_{n} \psi^{i}\right)=P_{n} f, \quad t \in[0, T] \\
v_{n}^{l}(0)=P_{n} x
\end{array}\right.
$$

with $i=1,2$. Set $\xi_{n}=v_{n}^{1}-v_{n}^{2}, \zeta=\psi^{1}-\psi^{2}$. Then

$$
\left\{\begin{array}{l}
\frac{d \xi_{n}}{d t}+A \xi_{n}+P_{n} B\left(v_{n}^{1}+P_{n} \psi^{1}, \xi_{n}+P_{n} \zeta\right)+P_{n} B\left(\xi_{n}+P_{n} \zeta, v_{n}^{2}+P_{n} \psi^{2}\right)=0 \\
\xi_{n}(0)=0
\end{array}\right.
$$

Therefore, after a few elementary manipulations,

$$
\begin{aligned}
\frac{1}{2} \frac{d}{d t}\left|\xi_{n}\right|^{2}+\left\|\xi_{n}\right\|^{2} \leqq & \left|\left\langle B\left(v_{n}^{1}+P_{n} \psi^{1}, \xi_{n}\right), P_{n} \zeta\right\rangle\right|+\left|\left\langle B\left(\xi_{n}+P_{n} \zeta, \xi_{n}\right), v_{n}^{2}+P_{n} \psi^{2}\right\rangle\right| \\
\leqq & C\left\|\xi_{n}\right\|\left|A^{\frac{1}{4}}\left(v_{n}^{1}+P_{n} \psi^{1}\right)\right|\left|A^{\frac{1}{4}} \zeta\right| \\
& +C\left\|\xi_{n}\right\|\left|A^{\frac{1}{4}}\left(\xi_{n}+P_{n} \zeta\right)\right|\left|A^{\frac{1}{4}}\left(v_{n}^{2}+P_{n} \psi^{2}\right)\right| \\
\leqq & \frac{1}{4}\left\|\xi_{n}\right\|^{2}+C\left|A^{\frac{1}{4}}\left(v_{n}^{1}+P_{n} \psi^{1}\right)\right|^{2}\left|A^{\frac{1}{4}} \zeta\right|^{2}+\frac{1}{4}\left\|\xi_{n}\right\|^{2} \\
& +C\left|A^{\frac{1}{4}} \xi_{n}\right|^{2}\left|A^{\frac{1}{4}}\left(v_{n}^{2}+P_{n} \psi^{2}\right)\right|^{2}+C\left|A^{\frac{1}{4}} \zeta\right|^{2}\left|A^{\frac{1}{4}}\left(v_{n}^{2}+P_{n} \psi^{2}\right)\right|^{2}
\end{aligned}
$$

Since

$$
\left|A^{\frac{1}{4}} \xi_{n}\right|^{2} \leqq\left|\xi_{n}\right|\left|A^{\frac{1}{2}} \xi_{n}\right|
$$

after some computations we have

$$
\begin{aligned}
\frac{1}{2} \frac{d}{d t}\left|\xi_{n}\right|^{2}+\frac{1}{4}\left\|\xi_{n}\right\|^{2} \leqq & C\left|A^{\frac{1}{4}}\left(v_{n}^{1}+\psi^{1}\right)\right|^{2}\left|A^{\frac{1}{4}} \zeta\right|^{2} \\
& +C\left|\xi_{n}\right|^{2}\left|A^{\frac{1}{4}}\left(v_{n}^{2}+\psi^{2}\right)\right|^{4}+C\left|A^{\frac{1}{4}} \zeta\right|^{2}\left|A^{\frac{1}{4}}\left(v_{n}^{2}+\psi^{2}\right)\right|^{2} .
\end{aligned}
$$

Using again (30) to estimate the terms $\left|A^{\frac{1}{4}} v_{n}^{i}\right|$ by means of (19), and applying Gronwall lemma, we finally obtain (27).

Remark. A.2. By the convergence results of Remark A.1, the bound analogous to (27) holds true for $\left|v^{1}-v^{2}\right|_{C([0, T] ; H)}$.

Estimate 4. Let us now consider only the case $R \in(0, \infty)$, and denote by $v_{n}(t ; x)$ the solution of Eq. (16). Let $U_{n}(t)$ be the differential of the mapping $x \mapsto v_{n}(t ; x)$ in the direction $h$ at point $x$, for given $x, h \in H$ :

$$
U_{n}(t)=D_{x} v_{n}(t ; x) \cdot h
$$


Set also

$$
u_{n}(t ; x)=v_{n}(t ; x)+P_{n} \psi(t)
$$

so that $U_{n}$ is also the differential of the mapping $x \mapsto u_{n}(t ; x)$ in the direction $h$ at point $x$. Thus $U_{n}$ satisfies

$$
\begin{aligned}
\frac{d U_{n}}{d t}+ & A U_{n}+\Theta_{R}^{\prime}\left(\left|u_{n}\right|_{D\left(A^{\frac{1}{4}}\right)}^{2}\right)^{2}\left\langle A^{\frac{1}{4}} u_{n}, A^{\frac{1}{4}} U_{n}\right\rangle B\left(u_{n}, u_{n}\right) \\
& +\Theta_{R}\left(\left|u_{n}\right|_{D\left(A^{\frac{1}{4}}\right)}^{2}\right)\left\{B\left(u_{n}, U_{n}\right)+B\left(U_{n}, u_{n}\right)\right\}
\end{aligned}
$$

We have

$$
\begin{aligned}
\frac{1}{2} \frac{d}{d t}\left|U_{n}\right|^{2}+\left\|U_{n}\right\|^{2} \leqq & 2\left|\Theta_{R}^{\prime}\left(\left|u_{n}\right|_{D\left(A^{\frac{1}{4}}\right)}^{2}\right)\right|\left|A^{\frac{1}{4}} u_{n}\right|\left|A^{\frac{1}{4}} U_{n}\right|\left|\left\langle B\left(u_{n}, u_{n}\right), U_{n}\right\rangle\right| \\
& +\Theta_{R}\left(\left|u_{n}\right|_{D\left(A^{\frac{1}{4}}\right)}^{2}\right)\left|\left\langle B\left(U_{n}, u_{n}\right), U_{n}\right\rangle\right| \\
\leqq & 2 C\left|\Theta_{R}^{\prime}\left(\left|u_{n}\right|_{D\left(A^{\frac{1}{4}}\right)}^{2}\right)\right|\left|A^{\frac{1}{4}} u_{n}\right|^{3}\left|A^{\frac{1}{4}} U_{n}\right|\left\|U_{n}\right\| \\
& +\Theta_{R}\left(\left|u_{n}\right|_{D\left(A^{\frac{1}{4}}\right)}^{2}\right)\left\|U_{n}\right\|\left|A^{\frac{1}{4}} u_{n}\right|\left|A^{\frac{1}{4}} U_{n}\right|
\end{aligned}
$$

Here we have used the equalities

$$
\begin{gathered}
\left\langle B\left(u_{n}, u_{n}\right), U_{n}\right\rangle=-\left\langle B\left(u_{n}, U_{n}\right), u_{n}\right\rangle, \\
\left\langle B\left(U_{n}, u_{n}\right), U_{n}\right\rangle=-\left\langle B\left(U_{n}, U_{n}\right), u_{n}\right\rangle
\end{gathered}
$$

and the estimates (following from Hölder inequality)

$$
\begin{aligned}
& \left|\left\langle B\left(u_{n}, U_{n}\right), u_{n}\right\rangle\right| \leqq C\left|A^{\frac{1}{4}} u_{n}\right|^{2}\left\|U_{n}\right\| \\
& \left|\left\langle B\left(U_{n}, U_{n}\right), u_{n}\right\rangle\right| \leqq C\left\|U_{n}\right\|\left|A^{\frac{1}{4}} u_{n}\right|\left|A^{\frac{1}{4}} U_{n}\right|
\end{aligned}
$$

Therefore, recalling that $\Theta_{R}$ has compact support in $[-R-1, R+1]$,

$$
\frac{1}{2} \frac{d}{d t}\left|U_{n}\right|^{2}+\left\|U_{n}\right\|^{2} \leqq C(R)\left|A^{\frac{1}{4}} U_{n}\right|\left\|U_{n}\right\|,
$$

where here and in the sequel $C(R)$ denotes a constant depending on $R$ (but not on $n, \psi, x, h, f)$. From the interpolation inequality $\left|A^{\frac{1}{4}} U_{n}\right| \leqq C\left|U_{n}\right|^{\frac{1}{2}}\left\|U_{n}\right\|^{\frac{1}{2}}$ and the Young inequality,

$$
\begin{aligned}
\frac{1}{2} \frac{d}{d t}\left|U_{n}\right|^{2}+\left\|U_{n}\right\|^{2} & \leqq C(R)\left|U_{n}\right|^{\frac{1}{2}}\left\|U_{n}\right\|^{\frac{3}{2}} \\
& \leqq \frac{1}{2}\left\|U_{n}\right\|^{2}+C(R)\left|U_{n}\right|^{2}
\end{aligned}
$$


From the Gronwall inequality we have now

$$
\left|U_{n}(t)\right|^{2} \leqq C(R)|h|^{2} \quad \forall t \in[0, T],
$$

and therefore, using again the previous inequality,

$$
\begin{aligned}
\int_{0}^{T}\left\|U_{n}(s)\right\|^{2} d s & \leqq|h|^{2}+C(R) \int_{0}^{T}\left|U_{n}(s)\right|^{2} d s \\
& \leqq C(R)|h|^{2}
\end{aligned}
$$

(having given different values to $C(R)$ ).

Acknowledgements. We thank A. Debussche and D. Gạtarek for some inspiring ideas.

\section{References}

1. Albeverio, S., Cruzeiro, A.B.: Global flow and invariant (Gibbs) measures for Euler and Navier-Stokes two dimensional fluids. Commun. Math. Phys. 129, 431-444 (1990)

2. Bensoussan, A., Temam, R.: Equations stochastiques du type Navier-Stokes. J. Funct. Anal. 13, 195-222 (1973)

3. Chojnowska-Michalik, A., Goldys, B.: Existence, uniqueness and invariant measures for stochastic semilinear equations in Hilbert spaces. Preprint, 1993

4. Cruzeiro, A.B.: Solutions et mesures invariantes pour des equations stochastiques du type Navier-Stokes. Expositiones Mathematicae 7, 73-82 (1989)

5. Da Prato, G., Debussche, A., Temam, R.: Stochastic Burger equation. Nonlinear Anal. and Appl. 1 389-402 (1994)

6. Da Prato, G., Elworthy, K.D., Zabczyk, J.: Strong Feller property for stochastic semilinear equations. Stoch. Anal. Appl. 13(1), 35-46 (1995)

7. Da Prato, G., Gatarek, D.: Stochastic Burgers equation with correlated noise, Stochastics \& Stoch. Rep., to appear

8. Da Prato, G., Zabczyk, J.: Stochastic Equations in Infinite Dimensions. Cambridge: Cambridge Univ. Press, 1992

9. Doob, J.L.: Asymptotic properties of Markov transition probability. Trans. Am. Math. Soc. 64, 393-421 (1948)

10. Flandoli, F.: Dissipativity and invariant measures for stochastic Navier-Stokes equations. Nonlinear Anal. and Appl. 1, 403-423 (1994)

11. Flandoli, F., Gatarek, D.: Martingale and stationary solutions for stochastic Navier-Stokes equations. Probability Theory \& Rel. Fields, to appear

12. Fujita, H., Yashima, H.: Equations de Navier-Stokes Stochastiques non Homogenes et Applications. Scuola Normale Superiore, Pisa, 1992

13. Khas'minskii, R.Z.: Ergodic properties of recurrent diffusion processes and stabilization of the solutions to the Cauchy problem for parabolic equations. Theory of Prob. and its Appl. 5, 179-196 (1960)

14. Manthey, R., Maslowski, B.: Qualitative behaviour of solutions of stochastic reaction-diffusion equations. Stoch. Proc. and their Appl. 43, 265-289 (1992)

15. Maslowski, B.: On probability distributions of solutions of semilinear stochastic evolution equations. Stochastics 45, 17-44 (1993)

16. Monin, A.S., Yaglom, A.M.: Statistical Hydrodynamics, Cambridge, MA: MIT Press, 1977

17. Pazy, A.: Semigroups of Linear Operators and Applications to Partial Differential Equations. Berlin, Heidelberg, New York: Springer, 1983

18. Peszat, S., Zabczyk, J.: Strong Feller property and irreducibility for diffusions on Hilbert spaces. Preprint Inst. Math. Polish Academy of Sciences, n. 510, Warsaw, 1993

19. Seidler, J.: Ergodic behaviour of stochastic parabolic equations. Submitted to Mathemat. Nachrichten, 1993 
20. Stettner, L.: Remarks on ergodic conditions for Markov processes on Polish spaces. Bulletin Polish Acad. Sci., Math. 42 103-114 (1994)

21. Temam, R.: Infinite Dimensional Dynamics Systems in Mechanics and Physics. Berlin, Heidelberg, New York: Springer, 1988

22. Vishik, M.I.: Fursikov, A.V.: Mathematical Problems of Statistical Hydromechanics. Dordrecht: Kluwer, 1980

Communicated by Ya. G. Sinai 
